\title{
Depth of lesion model in children and adolescents with moderate to severe traumatic brain injury: use of SPGR MRI to predict severity and outcome
}

\author{
M A Grados, B S Slomine, J P Gerring, R Vasa, N Bryan, M B Denckla
}

\begin{abstract}
Objectives-The utility of a depth of lesion classification using an SPGR MRI sequence in children with moderate to severe traumatic brain injury (TBI) was examined. Clinical and depth of lesion classification measures of TBI severity were used to predict neurological and functional outcome after TBI.
\end{abstract}

Methods-One hundred and six children, aged 4 to 19 , with moderate to severe TBI admitted to a rehabilitation unit had an SPGR MRI sequence obtained 3 months afterTBI. Acquired images were analyzed for location, number, and size of lesions. The Glasgow coma scale (GCS) was the clinical indicator of severity. The deepest lesion present was used for depth of lesion classification. Speed of injury was inferred from the type of injury. The disability rating scale at the time of discharge from the rehabilitation unit (DRS1) and at 1 year follow up (DRS2) were functional outcome measures. Results - The depth of lesion classification was significantly correlated with GCS severity, number of lesions, and both functional measures, DRS1 and DRS2. This result was more robust for time 1 , probably due to the greater number of psychosocial factors impacting on functioning at time 2 . Lesion volume was not correlated with the depth of lesion model. In multivariate models, depth of lesion was most predictive of DRS1, whereas GCS was most predictive of DRS2.

Conclusions-A depth of lesion classification of TBI severity may have clinical utility in predicting functional outcome in children and adolescents with moderate to severe TBI.

(F Neurol Neurosurg Psychiatry 2001;70:350-358)

Keywords: traumatic brain injury; magnetic resonance imaging; Glasgow coma scale; lesion depth

Correspondence to:

Dr M A Grados, Kennedy Krieger Institute, Psychiatry Department, 4th Floor, 1750 East Fairmount, Baltimore, MD 21224, USA

mjgrados@mail.jhmi.edu

Received 14 January 2000 and in final form

23 October 2000

Accepted 23 October 2000

Closed head injury resulting in traumatic brain injury (TBI) is the leading cause of death or permanent disability in children and adolescents. ${ }^{1}$ Despite the high incidence, prevalence, and morbidity of TBI in paediatric populations few studies have examined specific neuroanatomical lesions in relation to measures of severity of injury or functional outcome after
TBI. Such an approach could provide a method to assess a child's vulnerability to neurological sequelae and adaptation after TBI. Specifically, the use of MRI to identify children at high risk for neuropsychiatric sequelae would aid in the development of therapeutic programmes and optimise the allocation of rehabilitation resources.

Whereas open head injuries usually result in focal damage to the cerebrum, closed head injury lesions can result in diffuse lesion foci that are difficult to localise either clinically or by imaging techniques. The main hallmarks of closed injury are cerebral contusions and diffuse axonal injury. ${ }^{2}$ Diffuse axonal injury is a known marker of TBI severity and contemporary imaging sequences allow the visualisation of diffuse axonal injury lesions. In non-human primates, corpus callosum and brain stem lesions have been used to classify severity of TBI. ${ }^{3}$ In humans, basal ganglia and thalamic lesions may be additional markers of severity. Modern imaging techniques have now established the neurological, psychiatric, and neuropsychological relevance of these brain areas in cognitive, emotional, and behavioural functioning in humans. ${ }^{4}$

A TBI depth of lesion model based on animal experimentation was postulated by Ommaya and Genarelli in $1974 .{ }^{6}$ The Ommaya-Genarelli model was applied by Adams et al to create a grade 1-3 classification of lesions in animal studies. ${ }^{3}$ In humans, deep lesions have been correlated with greater psychological impairment, ${ }^{7}$ persistent vegetative states after TBI in adults, ${ }^{8}$ and greater impairment of consciousness on hospital admission. $^{9}$

Although a clinical classification of TBI is available, ${ }^{10}$ no systematic approach has used neuroimaging data to predict outcome or disability in children in the chronic phase after TBI utilising the degree, type, and location of lesions. Given emergent brain imaging technologies, it is plausible to identify brain lesion sites that classify subgroups of vulnerable children with TBI.

It is hypothesised that children with deep brain lesions after TBI represent a subset of children highly vulnerable to neurological and functional disability. A classification based on and functional outcome. To this end, children and adolescents were classified into injury depth of lesion may thus predict neurological 
groups based on the deepest brain lesion present. Disability immediately after injury and at 1 year follow up were correlated with depth of lesion. An exploration of the mechanism of injury and depth of lesion was carried out. Number of lesions and size of lesions were also analyzed in relation to the depth of lesion severity groups. Finally, an additional model, classification by number of affected brain areas by subject, was also explored.

\section{Subjects and methods \\ PATIENTS \\ Enrollment}

One hundred and thirty patients who were consecutively admitted to the neurorehabilitation unit of a university affiliated hospital in Baltimore, MD between 1992 and 1997 were considered for the study. Ten patients had mild TBI, three had no MRI study completed, and 11 had no lesions visible on the scan in the target regions studied. Thus, 106 children, aged 4 to 19 with moderate to severe TBI were enrolled. Other exclusion criteria included previous admissions to hospital or emergency room visits for TBI, mild range of GCS score (13-15), premorbid mental retardation, documented child abuse, and premorbid CNS pathology such as seizure disorder. Most children were enrolled into the study in the first month after injury.

Brain MRI was performed within 3 months after injury to detect chronic lesions. The MRI SPGR images were analyzed as described below. A GCS score rating was obtained either on admission to the emergency room or at the scene. A disability rating scale (DRS) score was obtained at the time of discharge from the rehabilitation unit to produce the first DRS rating (DRS1). This rating was made by the child's occupational therapist. At 1 year follow up, a DRS score was obtained from the parent to produce a follow up rating (DRS2).

\section{MEASURES}

Demographic variables

Socioeconomic status - The Hollingshead four factor index of social status was used to calculate socioeconomic status. ${ }^{11}$ Values on this scale ranged from 0 to 66 , with higher values for higher status. Low, medium, and high socioeconomic status groups were formed as low 0-28, medium 29-38, high 39-66.

Parental education - Combined years of schooling for mother and father were used to obtain a score of parental education. Three levels of parental education were formed from the sum of the parent's education years. The categories were low ( $<24$ years), medium (24-32 years), and high ( $>32$ years) parental education.

Sex, age, and ethnicity-Demographic data were elicited directly from the parent or carer on initial interview. Age was broken down into three groups, 4-9 years, 10-14 years, and 16-20 years. Ethnic origin was noted as white, African-American, or other.

Clinical variables

Severity of clinical injury-The GCS was used to rate TBI severity. ${ }^{12}$ Only children with GCS scores of 3-12 (moderate to severe) were enrolled.

Termination of post-traumatic amnesia (PTA) was evaluated by administration of the children's orientation amnesia test (COAT). ${ }^{13}$ Type of injury - "High speed" injuries involved motor vehicle passengers (except where the use of seat belt was recorded), pedestrians, bicyclists, and motor cyclists. The "low speed" group included patients involved in falls, assaults, sports injuries, and passengers with seat belts.

Functional and motor disability-The DRS rates level of arousal, awareness, and cognitive ability for feeding, toileting, and grooming after TBI, on a scale with a maximum score of $30 .^{14}$ The DRS has not been measured in normal children and pertinent modifications were used, such as assessment of school and age appropriate independent functioning rather than job functioning. Physical functioning did not require major modifications for use in a paediatric population.

Brain lesion variables

A 1.5 Tesla GE scanner was used to obtain images. Most patients were trained to inhibit body movement during scanning through operant conditioning. ${ }^{15}$

Three MR image series were performed: (1) $\mathrm{T} 1$ weighted sagittal localising scan to identify the anterior commissure-posterior commissure (AC/PC) line for alignment of all oblique axial images; (2) axial spin density/T2 weighted scans with $5 \mathrm{~mm}$ thick contiguous slices based on images obtained from the vertex to the foramen magnum; (3) axial $\mathrm{T} 1$ weighted, 3D volumetric scans with $1.5 \mathrm{~mm}$ thick contiguous slices obtained from the vertex to the foramen magnum (spoiled gradient recalled echo in steady state (SPGR): 35; 45; 1: TR; TE; NEX; total scan time of 18 minutes).

Only the 3D T1 weighted images were used for this analysis. This sequence allows good definition of chronic injuries due to high spatial resolution and $\mathrm{T} 1$ and $\mathrm{T} 2{ }^{\star}$ contrast sensitivity. Images were displayed on a $1024 \times 1024$ pixel $3 \mathrm{D}$ workstation for evaluation by trained raters. Two independent reliable raters (CBQ and JPG) read each image. A senior board certified radiologist with subspecialty training in neuroradiology (RNB) adjudicated any disagreement on each of the readings. All lesions were manually outlined by the technologists. The clinician rater was not blinded to all cases. Both the neuroradiologist adjudicator and the senior technician rater were blinded to all cases. Consensus lesions and adjudicated lesions were used for the analysis.

Reliability of volumetric measurement and lesion detection reliability was evaluated on 10 randomly selected cases, in each case blindly by the two readers. Interrater reliability of volume measurements was determined by intraclass correlation coefficients (ICCs) of total lesion volume per patient reported by each reader. The ICC for adjusted total lesion volume per patient was 0.99 between reader 1 and reader 2 ; the ICC for the number of lesions per patient was 0.99 between reader 1 and reader 2 . There 
was no tendency for systematic bias between the two readers.

Focal injuries were defined as hyperintense or hypointense local signal abnormalities on 3D T1 weighted images. Only intra-axial abnormalities were considered, and these included diffuse axonal injury, cortical contusions, intracerebral haematomas, and infarcts. The vast majority of lesions were diffuse axonal injury. A proprietary software program, Allegro software, ${ }^{16}$ was used to compute lesion volumes. Volumetric data was then converted to the Talairach stereotaxic reference frame. ${ }^{17}$ Lesion locations were determined using a standardised 3D map of approximated brain regions according to positions and dimensions as defined in the Talaraich atlas.

The following variables were derived from the image analysis: location of lesions (frontal, temporal, corpus callosum, basal ganglia, thalamus, cerebellum, brain stem), number of lesions per scan, and lesion volumes for each of the brain areas studied. Cortical lesions not in frontotemporal areas were not employed in the analysis.

\section{MRI DEFINED LESION GROUPS}

Classification by depth of lesion

Initially, five patient groups were used in the analysis based on depth of lesion: frontotemporal, corpus callosum, basal ganglia, thalamus, and brain stem/cerebellum groups. The deepest lesion was the parameter of consideration to classify patients. The five classification groups by depth of lesion were:

- Frontal and/or temporal lesions only (FT)

- Frontal and/or temporal+corpus callosum (CC)

- Frontal and/or temporaltcorpus callosum+basal ganglia (BG)

- Frontal and/or temporaltcorpus callosum \pm basal ganglia+thalamus $(\mathrm{TH})$

- Frontal and/or temporaltcorpus callosum \pm basal ganglia \pm thalamus+brain stem/ cerebellum (BC).

Of the 106 patients, most belonged to the most superficial group (frontotemporal, $n=34$ ). The deepest lesion group had the next highest number of patients (brain stem-cerebellum, $\mathrm{n}=31$ ). Group 5 (BC) with brain stem/ cerebellum lesions, had a wide variance of lesion volumes. On further examination, this group contained a subgroup of patients that had only frontotemporal+brain stem/ cerebellum lesions; that is, no corpus callosum, basal ganglia or thalamic lesions or "subcortical” lesions. In fact, most of the cerebellum/

Table 1 Relative frequency and number of lesions in six depth of lesion groups

\begin{tabular}{|c|c|c|c|}
\hline \multicolumn{2}{|c|}{$\begin{array}{l}\text { Depth of lesion group according to deepest lesion } \\
\text { present }\end{array}$} & \multirow{2}{*}{$\begin{array}{c}\text { Frequency (\%) } \\
34(32.1)\end{array}$} & \multirow{2}{*}{$\begin{array}{l}\begin{array}{l}\text { Number of lesions } \\
\text { (mean (SD)) }\end{array} \\
7.4(6.0)^{\star}\end{array}$} \\
\hline 1 & Frontal \pm temporal (FT) & & \\
\hline 2 & $\begin{array}{l}\text { Frontal } \pm \text { temporal and } \\
\text { Brain stem } \pm \text { cerebellum }(\mathrm{FT} / \mathrm{BC})\end{array}$ & $6(5.7)$ & $11.1(7.4)$ \\
\hline 3 & Corpus callosum (CC) & $19(17.9)$ & $9.5(6.2)$ \\
\hline 4 & Basal ganglia (BG) & $9(8.5)$ & $11.2(9.2)$ \\
\hline 5 & Thalamus $(\mathrm{TH})$ & $13(12.3)$ & $10.4(8.0)$ \\
\hline 6 & Brain stem \pm cerebellum (BC) & $25(23.6)$ & $11.6(6.2)$ \\
\hline Total & & $106(100.0)$ & $14.2(7.6)$ \\
\hline
\end{tabular}

$\star$ Post hoc pairwise rank sum comparisons significant for group $1 v$ group $6(\mathrm{p}<0.05)$. brain stem lesions in this subgroup were relatively small. This subgroup was proposed as a sixth class $(\mathrm{FT} / \mathrm{BC})$ given the peculiarities of the lesion pattern that resembled the more superficial lesion group, the group with only frontotemporal lesions (FT). It is also recognised that the mechanism of lesion in this particular subgroup may be due to only contusions affecting frontotemporal regions and brain stem. The possibility of creating groups for only frontotemporal+thalamus lesions or only frontotemporal+basal ganglia lesions was negated by the few patients in each of these groups, two and three respectively, and the small or no impact on the analyses. The final depth of lesion group composition is shown in table 1 .

\section{Classification by number of affected areas}

A second classification of brain lesions was undertaken. The number of affected areas was taken into account, forming five groups according to the five areas studied as follows (cerebellar lesions were merged with brain stem lesions as only two patients had all six lesion areas affected):

- Frontotemporal lesions

- Corpus callosum lesions

- Basal ganglia lesions

- Thalamic lesions

- Brain stem-cerebellum lesions.

\section{STATISTICAL ANALYSIS}

Pearson's $\chi^{2}$ tests were used to examine the association between the demographic variables sex, age, ethnic group, parental education, socioeconomic status, and outcome (DRS1, DRS2). A Shapiro-Wilks test was applied to variables of interest to test for normality. None of the variables had a normal distribution. Two approaches were considered to address this problem: (a) A log transformation was applied to all lesion volumes; $(b)$ non-parametric tests were employed to assess main effects of depth of lesion groups. The Kruskal-Wallis test, a $\kappa$ sample generalisation of the two sample rank sum test, provides a non-parametric alternative to one way analysis of variance (ANOVA). Kruskal-Wallis tests were carried out to assess the effect of the depth of lesion classification in relation to GCS, number of lesions, size of lesions, type of injury, and DRS scores at discharge and at 1 year follow up (DRS1, DRS2). Post hoc corrected multiple comparisons were conducted for depth of lesion groups. We note that this last estimation is conservative, given the exploratory nature of this study. Finally, univariate and multivariate linear regression models were run predicting DRS1 and DRS2 by depth of lesion groups, GCS, age groups, number of lesions, and volume of lesions. A best fitting model was constructed with backward stepwise regression for prediction of DRS1 and DRS2. Final models were tested for collinearity and assumption of constant error variance by the variable inflation factor test and the Cook-Weisberg test for heteroscedacity, respectively. Statistical calculations were carried out using the STATA 6.0 statistical package. ${ }^{18}$ 
Table 2 Demographic characteristics in children and adolescents with moderate to severe closed head injury

\begin{tabular}{|c|c|c|c|c|}
\hline \multirow{4}{*}{$\begin{array}{l}\text { Characteristic } \\
\text { Sex }\end{array}$} & \multirow{3}{*}{$\begin{array}{l}\text { Variable } \\
\text { Male }\end{array}$} & \multirow{3}{*}{$\frac{n(\%)}{63(59)}$} & \multicolumn{2}{|c|}{ Median (interquartile range) } \\
\hline & & & DRS1 & \multirow{2}{*}{$\frac{D R S 2 f}{1(1-3)}$} \\
\hline & & & $(2-4)$ & \\
\hline & Female & $43(41)$ & $(2-5)$ & $1(1-3)$ \\
\hline \multirow[t]{3}{*}{ Ethnicity } & White & $44(42)$ & $(2-5)$ & $1(0-3)$ \\
\hline & African-American & $56(53)$ & $(2-5)$ & $3(1-3)^{\star}$ \\
\hline & Other & $6(5)$ & $(0-2)$ & $1(0-1)$ \\
\hline \multirow[t]{3}{*}{ Age $(y)$} & $4-9$ & $34(32)$ & $(2-6)$ & $2.5(1-3)$ \\
\hline & $10-14$ & 41 (39) & $(2-4)$ & $1(1-3)$ \\
\hline & $15-20$ & $31(29)$ & $(2-4)$ & $1(0-3)$ \\
\hline \multirow[t]{3}{*}{ Parental education $\ddagger$} & Low & $28(29)$ & $3.5(2-5)$ & $2(1-3)$ \\
\hline & Medium & $62(63)$ & $3(1-4)$ & $2(1-3)$ \\
\hline & High & $8(8)$ & $(2-3)$ & $0.5(0-1.5)$ \\
\hline \multirow[t]{3}{*}{ Socioeconomic status $\$} & Low & $36(34)$ & $(2-5)$ & $2(1-3)$ \\
\hline & Medium & $34(32)$ & $(1-4)$ & $1(1-3)$ \\
\hline & High & $36(34)$ & $(2-5)$ & $1(0.5-3.5)$ \\
\hline
\end{tabular}

${ }^{\star} \mathrm{p}<0.05$.

†Sex compared by rank sum Wilcoxon test; ethnicity, age, parental education, and socioeconomic status compared by Kruskal-Wallis test.

$\ddagger$ Parental education: low (less than $24 \mathrm{y}$ ), medium (24-32 y) and high (over $32 \mathrm{y}$ ) parental years of education; $\mathrm{n}=98$.

JSES based on Hollinghead's four factor index; low 0-28, medium 28-38, high 39-66.

\section{Results}

DEMOGRAPHICS

The relation of age, sex, and ethnic group to DRS1 and DRS2 was examined. There were significant effects only for ethnic group; African-American children had significantly higher, or more impaired, DRS scores at time 2. This result was confirmed by a multiple regression model predicting DRS 2 controlling for the effects of socioeconomic status and parental education. In this model, AfricanAmerican ethnicity was the only significant predictor of DRS2 (coefficient $=0.82, \mathrm{p}<0.05$ ). Demographic characteristics of the sample are shown in table 2.

\section{CLINICAL SEVERITY}

Given that $88.7 \%(n=94)$, of patients had a severe TBI (GCS 3-8), conclusions from this study are best generalised to this patient group.

There was a main effect for depth of lesion group by GCS (Kruskal-Wallis $p=0.04$ ). Corrected multiple pairwise comparisons showed a significant difference for group 1 (FT) $v$ group 6 (BC). A box plot graph of this relation is depicted in fig 1 .

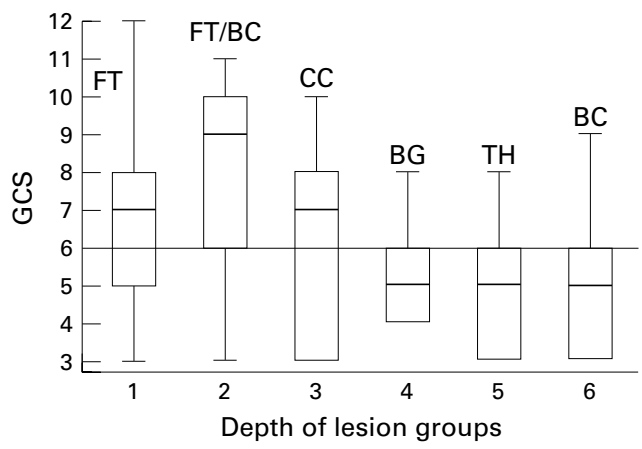

Figure 1 Glasgow coma scale (GCS) scores by six depth of lesion groups. $1 \mathrm{FT}=$ frontotemporal; 2 $F T / B C=$ frontotemporal $/$ brain stem-cerebellum; 3 $C C=$ corpus callosum; 4 BG=basal ganglia; 5 TH=thalamus; 6 BC=brain stem-cerebellum. Group median line shown, GCS=6.
Table 3 Type of injury in children and adolescents with moderate to severe traumatic brain injury

\begin{tabular}{lc}
\hline Type of injury & Frequency \\
\hline High speed injury: & 53 \\
Pedestrian & 16 \\
Automobile passenger & 11 \\
Bicycle & 7 \\
Automobile passenger without belt & 2 \\
Driver & 2 \\
Motor bike & 91 \\
Subtotal & \\
Low speed injury: & 3 \\
Sports & 4 \\
Fall & 4 \\
Assault & 4 \\
Automobile passenger with belt & 15 \\
Subtotal & 106 \\
Total & \\
\hline
\end{tabular}

NUMBER OF LESIONS

There was a mean number of 10.4 (SD 7.1) lesions in the 106 patients. A significant main effect was present in the number of lesions $\times$ lesion group analysis (Kruskal-Wallis $\mathrm{p}=0.01$ ). Corrected multiple pairwise comparisons showed a significant difference for number of lesions between group 1 (FT; mean 7.35) $v$ group 6 (BC; mean 14.24). Group relative frequency and number of lesions by group figures are provided in table 1.

VOLUME OF LESION

There was no significant main effect for $\log$ of total size of lesion $\times$ depth of lesion group (Kruskal-Wallis $\mathrm{p}=0.56$ ).

TYPE OF INJURY

Children sustained injuries in various ways (table 3 and fig 2). All lesion groups are represented equally in the high speed group $(n=95)$; but in the low speed group $(n=11)$, most patients had only superficial lesions (FT, FT/BC groups). Patients were divided into a group of only superficial lesions (FT, FT/BC groups) and another group consisting of all other lesions (CC, BG, TH, BC groups). Significant associations between the low speed group and superficial lesions (FT, FT/BC groups), and the high speed group and deeper lesions (CC, BG, TH, BC groups) are shown in tables 4 and 5 (Fisher's exact test $=0.003$ ).

MOTOR AND ADAPTATIONAL DISABILITY ON DISCHARGE FROM REHABILITATION UNIT (DRS1) Analysis by depth of lesion group was carried out for DRS at the time of discharge (DRS1) and at 1 year follow up (DRS2). There were nine patients for whom the DRS1 was not obtained.

The average DRS score at time of discharge from the unit (DRS1) was significantly higher (worse outcome) than the DRS score at 1 year follow up (DRS2) (3.4 (SD 2.7 v 1.9 (SD 1.7); paired Student's $t$ test, $t=6.35, \mathrm{p} \leqslant 0.001)$.

For DRS scores measured at the time of discharge from the rehabilitation unit (DRS1), there was a significant main effect for DRS1 $\times$ depth of lesion group (Kruskal-Wallis $\mathrm{p}=0.03)$. A box-whisker plot of DRS1 $\times$ depth of lesion group is presented in fig 3, showing 

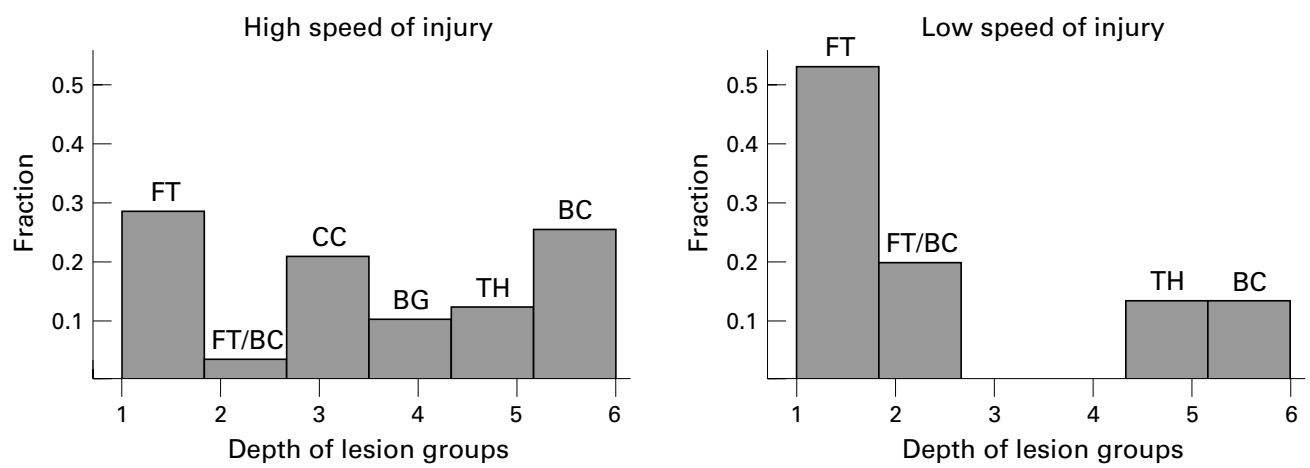

Figure 2 Depth of lesion groups and speed of injury (high speed injury $n=91$; low speed injury $n=15$ ). 1 $F T=$ frontotemporal; $2 \mathrm{FT} / \mathrm{BC}=$ frontotemporal $/$ brain stem-cerebellum; $3 \mathrm{CC}=$ corpus callosum; $4 \mathrm{BG}=$ basal ganglia; 5 TH=thalamus; 6 BC=brain stem-cerebellum.

worse outcome (higher DRS1) for greater depth of lesion.

DRS AT 1 YEAR FOLLOW UP (DRS2)

All patients had a follow up DRS score (DRS2, $\mathrm{n}=106)$. There was also a significant main effect for DRS $2 \times$ depth of lesion group (Kruskal-Wallis $\mathrm{p}=0.02$ ), but no post hoc pairwise comparisons were significant as seen in table 6 .

NUMBER OF AFFECTED AREAS AND FUNCTIONAL OUTCOME

A classification of patients by number of affected areas was used to form five groups. This classification resulted in 14 patients with only one affected brain area (13\%), 28 patients with two affected areas $(26 \%), 25$ patients with three affected areas (24\%), 23 patients with four affected areas $(22 \%)$, and 16 patients with five or more affected areas (16\%). Classification followed the number of affected brain areas, irrespective of the number of lesions in each area. Depth of lesion was not a factor in this classification scheme, although there is overlap given that patients with more affected areas also had deeper lesions. There was a main effect for this classification and GCS (KruskalWallis $\mathrm{p}=0.01$ ), DRS 1 (Kruskal-Wallis $\mathrm{p}=0.03$ ), and DRS2 (Kruskal-Wallis $\mathrm{p}=0.04$ ).

Table 4 Association between speed of injury and depth of lesion: six groups

\begin{tabular}{lllr}
\hline Depth of lesion group & $\begin{array}{l}\text { Low speed lesion } \\
n(\%)\end{array}$ & $\begin{array}{l}\text { High speed lesion } \\
n(\%)\end{array}$ & Total \\
\hline FT & $8(24)$ & $26(76)$ & 34 \\
FT/BC & $3(50)$ & $3(50)$ & 6 \\
CC & $0(0)$ & $19(100)$ & 19 \\
BG & $0(0)$ & $9(100)$ & 9 \\
TH & $2(15)$ & $11(85)$ & 13 \\
BC & $2(8)$ & $23(92)$ & 25 \\
\hline
\end{tabular}

Table 5 Association between speed of injury and depth of lesion: dichotomous groups

\begin{tabular}{lclc}
\hline Speed of injury & $\begin{array}{l}\text { Deep lesion presentt } \\
n(\%)\end{array}$ & $\begin{array}{l}\text { Superficial lesion only } \\
n(\%)\end{array}$ & $\begin{array}{l}\text { Total } \\
n(\%)\end{array}$ \\
\hline High speed of injury & $62(93.4)$ & $29(72.5)$ & $91(85.9)$ \\
Low speed of injury & $4(6.1)$ & $11(27.5)$ & $15(14.2)$ \\
Total & $91(100)$ & $15(100)$ & $106(100)$ \\
\hline
\end{tabular}

$(\mathrm{p}=0.003$, Fisher's exact test $)$

^Frontotemporal, or frontotemporal/brainstem-cerebellum (FT, FT/BC).

†Corpus callosum, basal ganglia, thalamus, brain stem-cerebellum (CC, BG, TH, BC).

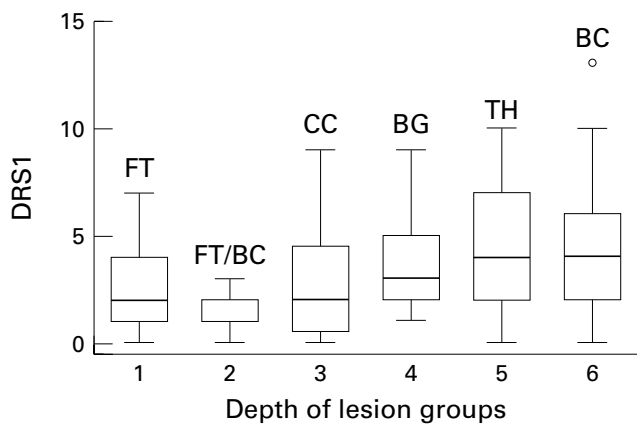

Figure 3 DRS scores at time of hospital discharge (DRS1) by depth of lesion groups in children and adolescents with moderate to severe traumatic brain injury. $1 \mathrm{FT}=$ frontotemporal $; 2 \mathrm{FT} / \mathrm{BC}=$ frontotemporal/brain stem-cerebellum; 3 CC=corpus callosum; $4 \mathrm{BG}=$ basal ganglia; $5 \mathrm{TH}=$ thalamus; $6 \mathrm{BC}=$ brain stem-cerebellum. $\mathrm{DRS} 1=$ disability rating scale score at hospital discharge (time 1).

The box plot for DRS $1 \times$ number of affected areas is shown in fig 4 .

UNIVARIATE AND MULTIVARIATE MODELS

Univariate linear regression analyses for both DRS1 and DRS2 showed that the depth of lesion classification was significantly predictive of worse DRS scores, both at time 1 and time 2 . The other significant predictor, GCS, was also a determinant of worse DRS at time 1 and time 2 .

Multivariate linear regression models were constructed to predict DRS1 and DRS2 scores by depth of lesion groups, volume of lesions, number of lesions, GCS, and age groups. A full multivariate model showed that only depth of lesion was predictive of DRS1. Both depth of lesion and GCS were predictive of DRS2, while controlling for volume of lesions, number of lesions, and age groups. The best fitting model for DRS1 included only depth of lesion groups and total lesion volume. For DRS2, the best fitting model included depth of lesion groups and GCS. The Cook-Weisberg test of heteroscedacity showed that constant error variance could not be confirmed for the DRS1 full model or best fitting model, but was present in the DRS2 full model and best fitting model. There was no evidence of collinearity between covariates in any of the models by the variance inflation factor test. 
Table 6 Univariate and multivariate linear regression models predicting DRS1 and DRS2 by depth of lesion, volume, and number of lesions, Glasgow coma scale (GCS) and age groups

\begin{tabular}{|c|c|c|c|c|}
\hline \multirow[b]{2}{*}{ Variable } & \multicolumn{2}{|c|}{ Univariate models } & \multicolumn{2}{|c|}{ Multivariate model } \\
\hline & Coefficient & $R^{2}$ & Coefficient & $R^{2}$ \\
\hline \multicolumn{5}{|c|}{ Models predicting DRS1: } \\
\hline Depth of lesion & $0.45^{\star \star}$ & 0.11 & $0.37^{\star}$ & 0.18 \\
\hline GCS & $-0.30^{\star}$ & 0.07 & -0.13 & \\
\hline Age groupt & -0.42 & 0.02 & -0.36 & \\
\hline Lesion volume & 0.24 & 0.03 & $0.26 \mathrm{t}$ & \\
\hline No of lesions & 0.07 & 0.03 & 0.01 & \\
\hline \multicolumn{5}{|c|}{ Models predicting DRS2: } \\
\hline Depth of lesion & $0.26^{\star \star}$ & 0.09 & $0.20^{\star}$ & 0.17 \\
\hline GCS & $-0.25^{\star \star}$ & 0.11 & $-0.18^{\star}$ & \\
\hline Age group & -0.26 & 0.01 & -0.15 & \\
\hline Lesion volume & 0.13 & 0.02 & $0.15 \mathrm{t}$ & \\
\hline No of lesions & 0.02 & 0.01 & -0.02 & \\
\hline
\end{tabular}

$\mathrm{t}=$ Trend, $0.05<\mathrm{p}<0.10 ;{ }^{\star} \mathrm{p}<0.05 ;{ }^{\star \star} \mathrm{p}<0.01$.

†Age groups: $4-9 \mathrm{y} ; 10-14 \mathrm{y} ; 15-20 \mathrm{y}$.

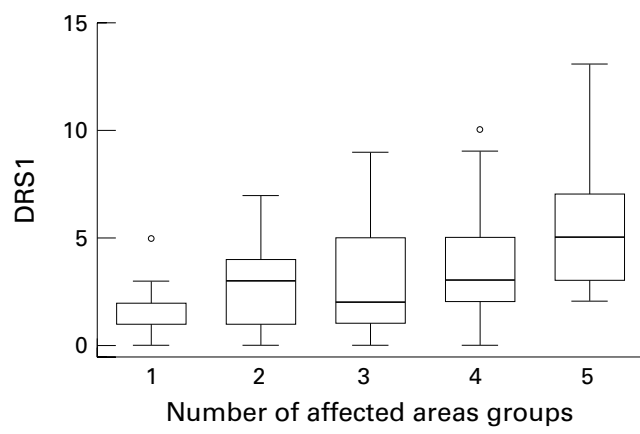

Figure 4 DRS scores at time of discharge (DRS1) by number of affected brain area groups in children and adolescents with moderate to severe traumatic brain injury. $D R S 1=$ disability rating scale score at hospital discharge

(time 1)

\section{Discussion}

A prospective series of 106 children and adolescents with moderate to severe TBI and identifiable brain lesions is presented. Intraaxial localisation of lesions after TBI was attained through a three dimensional SPoiled Grass (SPGR) MRI sequence producing five mutually exclusive depth of lesion groups. A sixth group, children with only frontotemporal and brain stem-cerebellum lesions, had both good clinical and prognostic indicators and emerged as a distinct non-overlapping group. When GCS severity scores, type of injury, total number of lesions, and motor/functional disability at time of discharge and at 1 year follow up were considered, a significant main effect for classification by six depth of lesion groups was present.

African-American children had a significantly worse functional outcome measure at 1 year follow up, independent of socioeconomic status and parental education. However, there were non-significant trends towards worse functional outcome at 1 year follow up in low strata of socioeconomic status and parental education, stressing the importance of psychosocial variables in outcome at 1 year follow up.

High speed injuries produced deeper lesions and low speed injuries produced more superficial lesions in this study. The high speed injury group reflected the occurrence of greater DAI in the corpus callosum, basal ganglia, thalamus, and brain stem/cerebellum. ${ }^{19}$ Levin et $a l^{20}$ have previously reported that speed of injury differentiates severity of lesions after TBI in patients with or without lesions. However, when only patients with visible lesions were examined in that study ( $55 \%$ of patients), the relation was no longer significant. It is possible that the greater sensitivity of the SPGR MRI technique in the current study sustains the greater correlation between depth of lesion and speed of injury.

The distinctive feature of this study is the use of SPGR MRI to detect brain lesion localisation, including diffuse axonal injury, in many children and adolescents with moderate to severe TBI. Strich first described diffuse axonal injury in 1956 as the diffuse "degeneration of the cerebral white matter" in post-traumatic dementia, and other early authors also described the "shearing injury of the white matter" in TBI by anatomicopathological ${ }^{2122}$ or CT studies. ${ }^{23}$ This lesion type is difficult to ascertain in the early phases of TBI, but appears in the chronic-greater than 3 weeksMRI used in this study. Acute studies of TBI have used proton magnetic resonance spectroscopy (MRS) in the region of the corpus callosum $^{24}$ and diffusion weighted $\mathrm{MRI}^{25}$ to detect diffuse axonal injury, but these techniques are not easily performed in clinical settings. Chronic MRI, as used in this study, can detect both haemorrhagic and non-haemorrhagic lesions, such as gliotic scars, with the ultimate resolution depending on several factors, including age of injury, presence of haemorrhage or blood breakdown products (haemosiderin), and type of sequence used. ${ }^{26}$ The greater power of the SPGR MRI sequence additionally lies in the use of $1.5 \mathrm{~mm}$ slices, providing significantly more resolution than the usual $5 \mathrm{~mm}$ slices.

Classification by depth of lesion has also been used by Levin et al both in adults ${ }^{27}$ and children, ${ }^{20}$ to examine the relation between depth of lesion and various severity and outcome variables. In these studies, a significant correlation was also found between GCS severity of injury and classification by depth of lesion, in 94 adults $^{27}$ and 251 children and adolescents from a large multicentre study. ${ }^{20}$

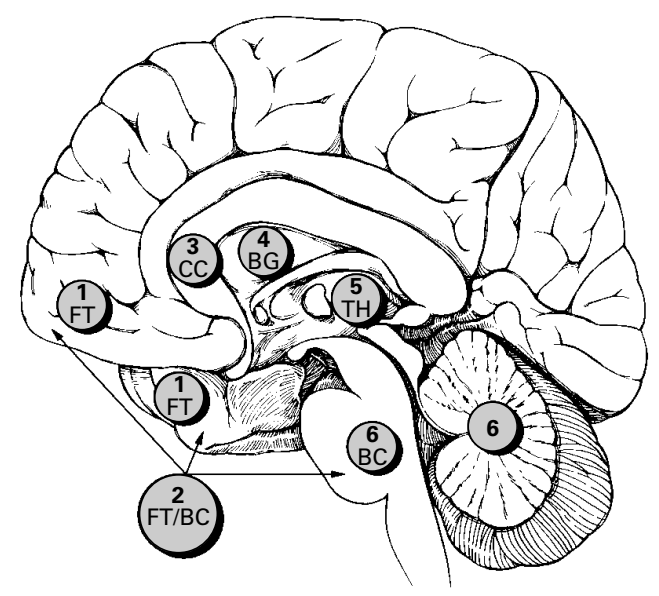

Figure 5 Classification model of TBI severity by six depth of lesion groups. $1 \mathrm{FT}=$ frontotemporal; 2 $F T / B C=$ frontotemporal/brain stem-cerebellum; 3 $C C=$ corpus callosum $; \quad B G=$ basal ganglia $; 5$ TH=thalamus; $6 \mathrm{BC}=$ brain stem-cerebellum. 
The range of severity studied in both of these reports was broader, and a larger proportion of patients with mild and moderate TBI were included. By comparison with previous studies, the children and adolescents examined in this study represent many moderate to severe children and adolescents with TBI with superficial and deep lesions specifically identified by a sensitive MRI technique. Six regions (fig 5) are more specific neuroanatomically than the three lesion groups reported by Levin et al. ${ }^{20} 27$

An additional classification scheme took into account the number of affected brain areas (range 1-5), irrespective of the total number of lesions. Patients with a greater number of lesioned areas had more impairment on functional outcome. The trend was stronger for DRS1, functional outcome at hospital discharge, and less striking for the 1 year follow up outcome measure. This pattern probably results from the greater weight of biological factors (lesions) affecting the initial outcome measure, whereas recovery at 1 year is plausibly also influenced by psychosocial factors. This classification also illustrates how groups with only two lesioned areas, such as the FT/BC group, would have better outcome overall than groups with three or more lesioned areas. Further exploration of this model is warranted.

As in previous studies, there was no direct relation between total size of lesion and depth of lesion groups - that is, lesion volume was not significantly associated with depth of lesion classification. This result replicates the results of Levin $e a^{20}{ }^{27}$ who found in two studies that lesion volume was not correlated with depth of lesion, using a different depth of lesion scheme. Deep lesions associated with functional impairment may have small volumes relative to superficial frontal contusions, making total lesion volume a possible poor predictor of TBI severity and functional outcome. However, when multivariate models are considered, total lesion volume contributes to some degree to predicting DRS outcome scores.

The depth of lesion model concurred with the use of GCS score in the assessment of clinical severity, with deeper lesions correlating with lower GCS scores. Although GCS scores have known value in predicting clinical outcome, they do not inform clinicians of the extent or site of lesions. Additional factors may complicate the use of a purely clinical measure such as GCS to predict outcome in TBI. For example, Quigley et al found an interaction between age at injury and GCS score for patients with severe head injury, with older patients showing worse outcome for the same GCS score. ${ }^{28}$ Another study found an interaction between GCS scores and types of brain lesion. ${ }^{29}$ In children with TBI, a low range of GCS scores has not always been predictive of poor outcome. ${ }^{30}$ Difficulty in applying GCS scores to young children includes developmental considerations such as limited use of language. Although there are alternative bedside prognostic factors for severity of TBI such as pupillary reaction, intracranial pressure monitoring, and brain stem auditory evoked potential, this information is not always available in the rehabilitation setting and the prognostic stability of these factors has not been conclusively established. ${ }^{31}$ Clearly, additional indices of severity and functional outcome other than GCS scores are needed for the optimal predictive power of functional outcome and disability in children afterTBI.

Finally, to our knowledge, there are no previous reports of specific neuroanatomical localisation of multiple diffuse axonal injury lesions using an SPGR MRI sequence in an extensive series of paediatric TBI. Previous use of T1 weighted MRI with an SPGR sequence has been used to produce an automatic atlas based volume estimation of brain regions, ${ }^{32}$ as well as to produce measurements of frontal lobes, ${ }^{33}$ cerebellum, ${ }^{34}$ and hippocampus/temporal lobes. ${ }^{35}$ SPGR MRI has also been used to evaluate intracranial tumours, ${ }^{36}$ cerebrovascular signals of flow, ${ }^{37}$ and fetal structures. ${ }^{38}$ Using an SPGR sequence, this study showed that number of lesions per patient was significantly different between depth of lesion groups with an average of 10.36 lesions per patient, 14.24 lesions in the more deeply affected group (BC) and 7.35 lesions in the least affected group (FT). These findings replicate those of Levin et $a l^{20}{ }^{27}$ who also found that total number of lesions was associated with greater depth of lesion, although the number of lesions detected was much smaller and only three depth of lesion groups were employed in that study. The use of number of lesions, as detected by SPGR MRI, may thus constitute a biologically plausible measure of TBI severity. A greater number of disconnections in neural tracts due to lesion foci may result in "disconnection syndromes" ${ }^{39}$ " Although a relation between disconnection syndromes and TBI sequelae cannot be currently established, disconnection syndromes have been postulated for dyslexia, ${ }^{40}{ }^{41}$ schizophrenia, ${ }^{42}$ and Alzheimer's disease, ${ }^{43}$ making it theoretically possible that a greater number of lesions may produce greater functional severity after TBI.

The DRS, a functional outcome and disability measure, was significantly different for depth of lesion groups, with greater disability found in the deeper lesion groups. This result was true for both time of discharge (DRS1) and at 1 year follow up (DRS2). As expected, there was also a significantly worse DRS measure at the time of hospital discharge (DRS1) than at 1 year follow up (DRS2), due to the influence of psychosocial factors in the rehabilitation process at 1 year. Multivariate models suggest that the depth of lesion classification is significantly predictive of DRS1, whereas DRS2 is impacted to a greater extent by the clinical measure GCS. When both depth of lesion and GCS are used in the model, the $R^{2}$ prediction parameter (explained variance of DRS) is improved. Also, the model for DRS2 was more stable due to constant error variance in this regression. Although possibly limited by its novel use in a paediatric population in this study, the DRS has been found to highly correlate with other scales used to assess functional outcome, such as the functional independence measure and the functional assessment measure. ${ }^{44}$ We conclude that based on DRS 
measures, especially DRS at time 2, the depth of lesion model predicts disability and functional outcome after TBI.

LIMITATIONS OF THE STUDY

Assumptions regarding brain variables are a limitation of this study. Although a neural model of severity of brain injury and functional outcome is described, some brain areas were not explored, such as the posterior cortical brain regions. However, these brain regions are not commonly lesioned in TBI. An additional limitation of this model is posed by the absence of separation between left and right sided lesions, limiting the study of the impact of laterality. Also, although it may be inferred that more superficial lesions are contusions and deeper lesions are diffuse axonal injury, no firm conclusions can be drawn about the "weight" of the type of lesion on the final outcome.

The speed on impact variable also required some assumptions given the data available. For example, when a child was a pedestrian or in a bicycle accident, it was assumed that this injury involved impact with a motor vehicle; however, these data were obtained from various sources including medical records and may not have been uniformly reported.

Functional outcome was measured by observer ratings through the DRS, posing natural limitations. The DRS was designed for adult populations; there is no current normative data for children. Although the DRS was modified for use with children in this study (for example, by substituting age appropriate levels of independence), it remains unclear how uninjured children would fare on this measure. The current analyses partially circumvented this limitation by comparing scores before and after DRS. Also, because the DRS was completed by a therapist at time 1 and through parental report at time 2, comparisons are subject to the variability inherent in the use of this reporting method.

The less robust correlation of the depth of lesion classification with DRS2, outcome at 1 year follow up, may be due to the fact that some lesions may have not been noted in the 3 month SPGR MRI. However, the use of $1.5 \mathrm{~mm}$ slices with this technique, decreases the possibility of discounting significant lesions.

Finally, the proposed models are supported by post hoc analyses driven by empirical subgroupings. For example, a particular empirical subgroup, the FT/BC group, had a unique good prognostic profile. By indirect inference, the three brain regions not containing lesions (corpus callosum, basal ganglia, and thalamus) might be "critical" lesion areas in outcome after TBI. Traditionally, brain stem injuries, in conjunction with DAI lesions in other areas or by themselves ${ }^{45}$ have been associated with poor prognosis. However, Bhatoe recently reported nine cases of primary brain stem injury with benign course and improved survival, paralleling our findings. ${ }^{46}$ The nature of the brain stem lesions in these cases needs to be further explored, possibly following. the distinction of Kampfl et al of primary and secondary brain stem injuries after TBI. ${ }^{8}$

\section{Conclusions}

A lesion based approach to increasing prognostic power predicting neurological and functional disability is supported by data on many children and adolescents with TBI. Clearly, future research on brain injury can profit from a lesion based approach that takes into account multiple and mutually exclusive brain lesion groups, such as depth of lesion or number of affected areas. Identification of lesions in the recovery phase of TBI may thus aid in the planning of rehabilitation treatment. Optimal allocation of health care resources to children with TBI who are at risk for poor outcome and their families would be possible.

This research was supported by: NIH grants K20 MH-00997 and M01RR00052.

1 Guyer B, Ellers B. Childhood injuries in the United States. American fournal of Diseases of Children 1990;144:649-52.

2 Cassidy JW. Neuropathology. In: Silver JM, Yudofsky SC, Hales RE, eds. Neuropsychiatry of traumatic brain injury. Washington, DC: American Psychiatric Press, 1994:43-79.

3 Adams JH. Head Injury. In: Adams JH, Duchen LW, eds. Greenfield's neuropathology. New York: Oxford University Press, 1992:106-52.

4 Alexander GE, DeLong MR, Strick PL. Parallel organization of functionally segregated circuits linking basal ganglia tion of functionally segregated circuits linking bas

5 Salloway S, Malloy P, Cummings JL. The neuropsychiatry of Salloway S, Malloy P, Cummings JL. The neuropsychiatry of
limbic and subcortical disorders. Washington, DC: American Psychiatric Press, 1997.

6 Ommaya AK, Gennarelli TA. Cerebral concussion and traumatic unconsciousness. Brain 1974;97:633-54.

7 Wilson JTL, Wiedmann KD, Hadley DM, et al. Early and late magnetic resonance imaging and neuropsychological outcome after head injury. F Neurol Neurosurg Psychiatry 1988;51:391-6.

8 Kampfl A, Franz G, Aichner F, et al. The persistent vegetative state after closed head injury: clinical and magnetic resonance imaging findings in 42 patients. 7 Neurosurg 1998;88:809-16.

9 Jenkins A, Teasdale G, Hadley MDM, et al. Brain lesions detected by magnetic resonance imaging in mild and severe head injury. Lancet 1986;ii:445-6.

10 Teasdale G, Teasdale E, Hadley D. Computed tomographic and magnetic resonance imaging classification of head and magnetic resonance imaging classification

11 Hollingshead AB. Four factor index of social status. New Haven, CT: Yale University Department of Sociology, 1975.

12 Teasdale G, Jennett B. Assessment of coma and impaired consciousness: a practical scale. Lancet 1974;ii:81-4.

13 Ewing-Cobbs L, Levin HS, Fletcher JM, et al. The children's orientation and amnesia test: relationship to severity of acute head injury and to recovery of memory. Neurosurgery 1990;27:683-91.

14 Rappaport M, Hall KM, Hopkins K, et al. Disability rating scale for severe head trauma: coma to community. Arch Phys Med Rehabil 1982;63:118-23.

15 Slifer K, Cataldo MF, Cataldo MD, et al. Behavior analysis of motor control for pediatric neuroimaging. F Appl Behav Anal 1993;26:469-70.

16 ISG Technologies. Allegro software. Toronto, Canada: ISGT, 1995.

17 Talairach J, Tournoux P. Co-planar stereotaxic atlas of the human brain: 3-D proportional system. New York: Thieme Medical, 1988.

18 Stata Corporation. Stata (6.0). College Station, Texas: Stata, 1999.

19 Alexander MP. Traumatic brain injury. In: Benson DF, Blumer E, eds. Psychiatric aspects of neurological disease. Vol II. New York: Grune and Stratton, 1982:219-48.

20 Levin HS, Mendelsohn D, Lilly MA, et al. Magnetic resonance imaging in relation to functional outcome of pediatric closed head injury: a test of the OmmayaGennarelli model. Neurosurgery 1997;40:432-40.

21 Strich SJ. Shearing of nerve fibres as a cause of brain damage due to head injury. Lancet 1961;ii:443-8.

22 Adams JH, Mitchell DE, Graham DI, et al. Diffuse brain damage of immediate impact type. Brain 1977;100:489502.

23 Zimmerman RA, Larissa T, Bilajnuk LT, et al. Computed tomography of shearing injuries of the cerebral white mattomography of shearing injuries

24 Cecil KM, Hills EC, Sandel ME, et al. Proton magnetic resonance spectroscopy for detection of axonal injury in the splenium of the corpus callosum of brain-injured patients. f Neurosurg 1998;88:795-801. 
25 Alsop DC, Murai H, Detre JA, et al. Detection of acute pathologic changes following experimental traumatic brain injury using diffusion-weighted magnetic resonance imaging. F Neurotrauma 1996;13:515-21.

26 Parizel PM, Ozsarlak, Van Goethem JW, et al. Imaging findings in diffuse axonal injury after closed head trauma. Eu Radiol 1998;8:960-5.

27 Levin HS, Williams D, Crofford MJ, et al. Relationship of depth of brain lesions to consciousness and outcome after closed head injury. F Neurosurg 1988;69:861-6.

28 Quigley MR, Vidovich D, Cantella D, et al. Defining the limits of survivorship after very severe head injury. Fournal of Trauma 1997;42:7-10.

29 Katz DI, Alexander MP. Traumatic brain injury. Predicting course of recovery and outcome for patients admitted to rehabilitation. Arch Neurol 1994;51:661-70.

30 Lieh-Lai MW, Theodorou AA, Sarnaik AP, et al. Limitations of the Glasgow coma scale in predicting outcome in children with traumatic brain injury. f Pediatr 1992;120:195-9.

31 Krieger D, Adams HP, Schwarz S, et al. Prognostic and clinical relevance of pupillary responses, intracranial pressure monitoring, and brainstem auditory evoked pressure monitoring, and brainstem auditory evoked potentials in comatose patients with acute sup

32 Andreasen NC, Rajarethinam R, Cizadlo T, et al. Automatic atlas-based volume estimation of human brain regions from MR images. F Comput Assist Tomogr 1996;20:98-106.

33 Aylward EH, Augustine A, Li Q, et al. Measurement of frontal lobe volume on magnetic resonance imaging scans. Psychiatry Res 1997;75:23-30.

34 Paradiso S, Andreasen NC, O'Leary DS, et al. Cerebellar size and cognition: correlations with IO, verbal memory and motor dexterity. Neuropsychiatry Neuropsychol Behav Neurol 1997;10:1-8.

35 Torres IJ, Flashman LA, O'Leary DS, et al. Lack of an association between delayed memory and hippocampal and temporal lobe size in patients with schizophrenia and healthy controls. Biol Psychiatry 1997;42:1087-96.
36 Rand S, Maravilla KR. Uses and limitations of spoiled gradient-refocused imaging in the evaluation of suspected intracranial tumors. Top Magn Reson Imaging 1992;4: 7-16.

37 Shogry ME, Elster AD. Cerebrovascular enhancement in spoiled GRASS (SPGR) images: comparison with spin-echo technique. f Comput Assist Tomogr 1992;16:4853.

38 Colletti PM. Computer-assisted imaging of the fetus with magnetic resonance imaging. Comput Med Imaging Graph 1996;20:491-6.

39 Povlishock JT, Christman CW. The pathobiology of traumatically induced axonal injury in animals and humans: a review of current thoughts. F Neurotrauma 1995; 12:555-64.

40 Horwitz B, Rumsey JM, Donohue BC. Functional connectivity of the angular gyrus in normal reading and dyslexia. Proc Natl Acad Sci USA 1998;95:8939-44.

41 Paulesu E, Frith U, Snowling M, et al. Is developmental dyslexia a disconnection syndrome? Evidence from PET scanning. Brain 1996;119:143-57.

42 Friston KJ. Theoretical neurobiology and schizophrenia. $\mathrm{Br}$ Med Bull 1996;52:644-55.

43 Terry RD. The pathogenesis of Alzheimer disease: an alternative to the amyloid hypothesis. $\mathcal{F}$ Neuropathol Exp Neurol 1996;55:1023-5.

44 Hall KM, Hamilton BB, Gordon WA. Characteristics and comparisons of functional assessment indices: disability rating scale, functional independence measure, and functional assessment measure. $\mathcal{F}$ Head Trauma Rehabil 1993;8: 60-74.

45 Kim RC, Fagin K, Choi BH. Prolonged survival after severe traumatic injury limited to the brainstem. Surg Neurol 1985;23:525-8.

46 Bhatoe HS. Primary brainstem injury: benign course and improved survival. Acta Neurochir (Wien) 1999;141:515-9. 